\title{
OPEN BRCA1 and BRCA2 genes mutations among high risk breast cancer patients in Jordan
}

\author{
Munir Abu-Helalah ${ }^{1,2}$, Belal Azab ${ }^{3,4}$, Rasmi Mubaidin ${ }^{5}$, Dema Ali ${ }^{3}$, Hanan Jafar ${ }^{3,6}$, \\ Hussam Alshraideh ${ }^{7,8}$, Nizar Drou ${ }^{9}$ \& Abdalla Awidi ${ }^{3,10 凶}$
}

Familial breast cancer is estimated to account for $15-20 \%$ of all cases of breast cancer. Surveillance for familial breast cancer is well-established world-wide. However, this service does not exist in Jordan, due to the scarcity of information with regard to the genetic profiling of these patients, and therefore lack of recommendations for policy-makers. As such, patients with very strong family history of breast or ovarian cancers are not screened routinely; leading to preventable delay in diagnosis. Whole coding sequencing for BCRA1/BCRA2 using next-generation sequencing (NGS)/Ion PGM System was performed. Sanger sequencing were then used to confirm the pathogenic variants detected by NGS. In this study, 192 breast cancer patients (and 8 ovarian cancer cases) were included. The prevalence of recurrent pathogenic mutations was $14.5 \%$, while the prevalence of newly detected mutations was 3.5\%. Two novel pathogenic mutations were identified in BRCA2 genes. The common mutations in the Ashkenazi population used for screening may not apply in the Jordanian population, as previously reported mutations were not prevalent, and other new mutations were identified. These data will aid to establish a specific screening test for BRCA $1 / B R C A 2$ in the Jordanian population.

Breast cancer is the most common cancer in females worldwide contributing to $25.4 \%$ of the total number of new cancer cases among women in 2018. Worldwide, more than two million new cases are diagnosed each year and nearly 600,000 women die from breast cancer each year ${ }^{1}$. According to the Jordanian National Cancer Registry report of the year 2015, there were 1145 female breast cancer cases accounting for $20.6 \%$ of all newly-diagnosed cancer cases and $39.4 \%$ of the cancers in females ${ }^{2}$.

Breast cancer at an early age is more likely to be associated with an increased familial risk as hereditary predisposition is estimated to cause about $5-10 \%$ of all breast cancers ${ }^{3}$. Nearly $50 \%$ of all hereditary breast cancer cases are due to germline mutations in the BRCA1/BRCA2 genes and are associated with early-onset breast cancer ${ }^{4}$. The cumulative risk estimates for developing breast cancer by age 80 are $72 \%$ for $B R C A 1$ carriers and $69 \%$ for BRCA2 carriers ${ }^{4}$.

Nearly 1650 mutations and sequence variants have been reported in the $B R C A 1$ gene and 1730 in $B R C A 2^{5}$. $B R C A 1$ and $B R C A 2$ are both tumor suppressor genes that play a role in DNA repair. The $B R C A 1$ gene plays also a role in checkpoint control. Mutations in either gene are associated with an autosomal dominant inherited form of breast cancer ${ }^{6,7}$.

Several studies in the Arab world were done to identify the mutations in BRCA1/BRCA2 genes and to assess prevalence of known mutations ${ }^{8-12}$. The main limitations for the above studies have been the small number of participants and the uncontrolled selection criteria. A recent study conducted in Jordan showed that BRCA1/ $B R C A 2$ variants are common among Jordanian breast cancer patients as $27 \%$ of high-risk breast cancer patients had pathogenic or likely pathogenic variants in BRCA1 or BRCA2 genes 9

Recently, a comprehensive assessment of the mutational spectrum of BRCA1/BRCA2 in the Middle East, North Africa, and Southern Europe was published ${ }^{13}$. Laitamn et al. reported recurring pathogenic sequence variants (PSVs) identifying 232 PSVs and 239 PSVs in BRCA1 and BRCA2, respectively. This comprehensive data

\footnotetext{
${ }^{1}$ Department of Public Health, Faculty of Medicine, Mutah University, Karak, Jordan. ${ }^{2}$ Faculty of Medicine, Al-Faisal University, Riyadh, Kingdom of Saudi Arabia. ${ }^{3}$ Cell Therapy Center, The University of Jordan, Amman 11942, Jordan. ${ }^{4}$ Department of Pathology, School of Medicine, The University of Jordan, Amman, Jordan. ${ }^{5}$ Radiation Therapy Department, Al-Bashir Hospital, Ministry of Health, Amman, Jordan. ${ }^{6}$ Department of Anatomy and Histology, School of Medicine, The University of Jordan, Amman, Jordan. ${ }^{7}$ Industrial Engineering Department, University of Science and Technology, Irbid, Jordan. ${ }^{8}$ Industrial Engineering Department, American University of Sharjah, Sharjah, UAE. ${ }^{9}$ NYU Abu Dhabi Center for Genomics and System Biology, Abu Dhabi, UAE. ${ }^{10}$ Department of Medicine, School of Medicine, The University of Jordan, Amman, Jordan. ${ }^{\circledR}$ email: abdalla.awidi@gmail.com
} 
will lead to directed testing of high-risk subpopulations, and therefore earlier diagnosis and superior management leading to better outcomes. However, there is no founder mutation(s) in BRCA1/BRCA2 in the Jordanian population that could be used for early genetic screening.

In this study, the authors evaluated the potential contribution and the frequency of mutations in the $B C R A 1 / B C R A 2$ genes in selected Jordanian patients with breast and ovarian cancers, identified based on NICE criteria for high risk of familial cancer, through performing whole coding sequence for BCRA1/ BCRA2 with the purpose of providing baseline data for establishment of familial cancer services in Jordan.

\section{Materials and methods}

Ethical approval and consent to participate. The study was approved by the Institutional Review Boards and Ethics Committees at Mutah University and the Central Ethics Committee at the Ministry of Health, Jordan. All included patients were informed about the objective and protocol of the study and gave written informed consent in accordance with the Declaration of Helsinki prior to undergoing any procedures.

Study population. This prospective cross-sectional study was conducted et al. Bashir Hospital, Amman, Jordan. All Jordanian breast cancer patients referred to this hospital during the study period were under surveillance for familial breast or ovarian cancer according to a uniform selection criterion (see below).The U.K. National Institute for Health and Care Excellence (NICE), November 2013 Clinical guidelines for identification of patients with high risk for familial breast/ovarian cancer who required referral for testing in a specialist genetics clinic were used for identifying study participants and labelling them as 'high risk for familial cancer ${ }^{14}$.

Patients files were reviewed for clinical and pathology data such as stage, grade, site, laterality, oestrogen receptor status, HER2 (or HER2 neu) positive status, etc.

Sample collection and genomic DNA isolation. Peripheral blood samples were drawn from study participants (192 breast cancer patients and 8 ovarian cancer cases) and sent for analysis at the Cell Therapy Centre at Jordan University. Genomic DNA was extracted from $5 \mathrm{ml}$ EDTA tubes using QIAprep Spin Miniprep Kit according to manufacturer's recommendations, and quantified using Nano Drop Spectrophotometer (Thermo Fisher Scientific).

Next-generation sequencing and variant analysis. BRCA1/BRCA2 library preparation and Ion PGM next-generation sequencing. The coding exons and flanking intronic regions of the BRCA1/BRCA2 genes were amplified using Ion AmpliSeq Library Kit 2.0 (Life Technologies) and Ion AmpliSeq BRCA1/BRCA2 Panel (Life Technologies). The whole process was performed following the manufacturer's instructions with minor modifications as previously described ${ }^{15,16}$. Each library was barcoded using Ion Xpress Barcode Adapters kit (Life Technologies) and its concentration was determined with Ion Library TaqMan Quantitation Kit (Life Technologies). For template preparation, emulsion PCR and enrichment were carried out using Ion OneTouch 2 system and the Ion OneTouch ES Instrument (Life Technologies) with Ion PGM Hi-Q View OT2 Kit (Life Technologies). An average of six prepared barcoded samples were loaded into a single Ion 316 Chip v2 (Life Technologies) per sequencing run. The sequencing reactions were performed in the Ion torrent PGM System (Life Technologies) using sequencing Ion PGM Hi_Q view sequencing kit (Life Technologies). The generated data on Ion PGM were processed through on Torrent Suite, version 5.0.4, running on a local server (Life Technologies). The VCF files were uploaded to Ion Reporter (Life Technologies) and annotated for single-nucleotide variants (SNV), insertions and deletions (indels), and splice site changes. Reads were visualized with the Integrative Genomics Viewer (IGV) and Alamut (Interactive Biosoftware, Rouen, France).

Evaluation of the variants clinical significance. Variants that are clearly classified in the Breast Cancer Information Core (BIC) database, Human Gene Mutation Database Pro (HGMD pro) and ClinVar databases, and not previously described as pathogenic in the literature were evaluated based on the guidelines and standards for interpretation of sequence variants recommended by the American College of Medical Genetics and Genomics (ACMG) ${ }^{17}$.

DNA Sanger sequencing. Sanger sequencing was performed for validating selected candidate variants identified by NGS. Primers flanking the candidate loci were designed using Primer 3.0 and synthesized by IDT, CA, USA (Supplementary table 1). PCR amplification was performed using Platinum PCR SuperMix (Invitrogen, CA, USA). Direct automated sequencing for the purified PCR products was performed on an ABI 3500 Capillary DNA Sequencer using the BigDye Terminator Cycle Sequencing kit (Applied Biosystems, CA, USA). The sequence chromatograms were visualized by Sequencing Analysis SeqA software (Applied Biosystems) and analyzed by Chromas Pro software (Technolysium Ltd., South Brisbane, Australia).

Statistical analysis. Collected Data was analyzed using R Statistical Computing Software version 3.4.3 (R Foundation for Statistical Computing, Vienna, Austria). Descriptive statistics including means and standard deviations are reported for numerical patient characteristics while frequencies and percentages are reported for categorical characteristics. Predictors of $B R C A 1$ and $B R C A 2$ mutations were identified through multiple linear regression with stepwise selection. A significance level of 0.05 is used throughout the analysis. 


\begin{tabular}{|c|c|c|c|c|}
\hline Value & $\mathbf{N}$ & Raw \% & Valid \% & Cumulative \% \\
\hline \multicolumn{5}{|c|}{ Primary site } \\
\hline Breast & 192 & 96 & 96 & 96 \\
\hline Ovaries & 8 & 4 & 4 & 100 \\
\hline \multicolumn{5}{|c|}{ Site of cancer } \\
\hline Bilateral & 15 & 7.5 & 7.5 & 7.5 \\
\hline Left & 92 & 46 & 46 & 53.5 \\
\hline Right & 93 & 46.5 & 46.5 & 100 \\
\hline \multicolumn{5}{|c|}{ Stage at diagnosis for breast cancer } \\
\hline I & 46 & 23 & 23 & 23 \\
\hline II & 64 & 32 & 32 & 55 \\
\hline III & 34 & 17 & 17 & 72 \\
\hline IV & 56 & 28 & 28 & 100 \\
\hline \multicolumn{5}{|l|}{ Metastasis } \\
\hline No & 148 & 74 & 74 & 74 \\
\hline Yes & 52 & 26 & 26 & 100 \\
\hline \multicolumn{5}{|c|}{ Recurrence since baseline } \\
\hline No & 194 & 97 & 97 & 97 \\
\hline Yes & 6 & 3 & 3 & 100 \\
\hline \multicolumn{5}{|c|}{ Laterality for breast cancer } \\
\hline $\mathrm{Bi}$ & 16 & 8 & 8 & 8 \\
\hline Uni & 184 & 92 & 92 & 100 \\
\hline \multicolumn{5}{|c|}{ Tumor size at histological examination } \\
\hline$(<2 \mathrm{~cm})$ & 34 & 17 & 17 & 17 \\
\hline$(>=2 \mathrm{~cm})$ & 166 & 83 & 83 & 100 \\
\hline \multicolumn{5}{|c|}{ Extent of disease } \\
\hline Distant & 53 & 26.5 & 26.6 & 26.6 \\
\hline Local & 41 & 20.5 & 20.6 & 47.2 \\
\hline Regional & 105 & 52.5 & 52.8 & 100 \\
\hline \multicolumn{5}{|c|}{ Oestrogen Receptors status } \\
\hline Negative & 7 & 3.5 & 3.6 & 3.6 \\
\hline Positive & 29 & 14.5 & 14.9 & 18.6 \\
\hline Unknown & 158 & 79 & 81.4 & 100 \\
\hline \multicolumn{5}{|l|}{ ER } \\
\hline Negative & 37 & 18.5 & 19.1 & $\mid 19.1$ \\
\hline Positive & 139 & 69.5 & 71.6 & 90.7 \\
\hline Unknown & 18 & 9 & 9.3 & 100 \\
\hline \multicolumn{5}{|l|}{ PR } \\
\hline Negative & 46 & 23 & \begin{tabular}{|l|}
23.7 \\
\end{tabular} & 23.7 \\
\hline Positive & 133 & 66.5 & 68.6 & 92.3 \\
\hline Unknown & 15 & 7.5 & \begin{tabular}{|l|}
7.7 \\
\end{tabular} & 100 \\
\hline \multicolumn{5}{|l|}{ Hert-Neu } \\
\hline Negative & 129 & 64.5 & 66.8 & 66.8 \\
\hline Positive & 49 & 24.5 & 25.4 & 92.2 \\
\hline Unknown & 15 & 7.5 & 7.8 & 100 \\
\hline \multicolumn{5}{|l|}{ E cadherin } \\
\hline Negative & 18 & 9 & 9.3 & 9.3 \\
\hline Positive & 16 & 8 & 8.2 & 17.5 \\
\hline Unknown & 160 & 80 & 82.5 & 100 \\
\hline
\end{tabular}

Table 1. Clinical characteristics of study participants.

\section{Results}

Participants. Of the 200 patients included in the study; 192 of them had breast cancer as the primary cancer site, while the remaining eight cases ovarian cancer as the primary site. $7.5 \%$ of them (15 patients) had bilateral breast cancer and $3 \%$ (6 patients) had recurrent cancer. The mean age of the study population was $43.6 \pm 2.7$ with an age interval of 22 to 70 . Regarding clinical characteristics of participants (Table 1), $45 \%$ of patients were diagnosed at stage III or IV, while $32 \%$ of them were diagnosed at stage II. $26.5 \%$ had distant and $52.5 \%$ had regional 


\begin{tabular}{|c|c|c|}
\hline Category & Number of patients & Prevalence \\
\hline \multicolumn{3}{|l|}{ Recurrent mutations } \\
\hline BRCA1 Positive & 15 & $7.50 \%$ \\
\hline BRCA2 Positive & 14 & $7.00 \%$ \\
\hline BRCA1 or BRCA2 Positive & 29 & $14.50 \%$ \\
\hline \multicolumn{3}{|c|}{ Possible (recurrent and novel) mutations } \\
\hline BRCA1 Positive & 7 & $3.50 \%$ \\
\hline BRCA2 Positive & 14 & $7.00 \%$ \\
\hline BRCA1 or BRCA2 Positive & 21 & $10.50 \%$ \\
\hline \multicolumn{3}{|c|}{ Recurrent and novel (VUS and pathogenic) mutations } \\
\hline BRCA1 Positive & 15 & $7.50 \%$ \\
\hline BRCA2 Positive & 21 & $10.50 \%$ \\
\hline BRCA1 or BRCA2 Positive & 36 & $18.00 \%$ \\
\hline
\end{tabular}

Table 2. Prevalence of mutations pathogenic and VUS mutations.

\begin{tabular}{|c|c|c|c|c|c|c|c|c|c|c|c|c|c|c|c|}
\hline \multicolumn{4}{|l|}{ Variant } & \multirow[b]{2}{*}{$\begin{array}{l}\text { Case Freq/ } \\
\text { Zygosity }\end{array}$} & \multirow[b]{2}{*}{$\begin{array}{l}\text { Type of } \\
\text { cancer }\end{array}$} & \multicolumn{3}{|c|}{ Mutation Database } & \multirow[b]{2}{*}{ dbSNP ID } & \multicolumn{4}{|c|}{ Protein Prediction } & \multirow[b]{2}{*}{$\begin{array}{l}\text { MAFgnomAD } \\
(\%)\end{array}$} & \multirow[b]{2}{*}{ Reference } \\
\hline $\begin{array}{l}\text { Exon/ } \\
\text { Intron }\end{array}$ & HGVS cDNA & HGVS aa & \begin{tabular}{|l|} 
Variant \\
Effect
\end{tabular} & & & ClinVar & BIC & \begin{tabular}{l|} 
HGMD(Accession \\
$\#)$
\end{tabular} & & SIFT & PolyPhen-2 & \begin{tabular}{|l|} 
Mutation \\
Taster
\end{tabular} & CONDEL & & \\
\hline \multicolumn{16}{|c|}{$B R C A 1$ : pathogenic variants } \\
\hline E18 & c. $5186 \mathrm{C}>\mathrm{A}$ & p.Ala1729Glu & Missense & $1(0.5 \%)$ Het & Breast & Pathogenic & CI & \begin{tabular}{|l} 
DM \\
CM950153
\end{tabular} & rs28897696 & \begin{tabular}{|l|}
$\begin{array}{l}\text { Delete- } \\
\text { rious }\end{array}$ \\
\end{tabular} & \begin{tabular}{|l|} 
Probably \\
damaging
\end{tabular} & $\begin{array}{l}\text { Disease } \\
\text { causing }\end{array}$ & Deleterious & 0.0004 & 21 \\
\hline E18 & c. $5158 \mathrm{C}>\mathrm{T}$ & p.Arg1720Trp & Missense & $1(0.5 \%)$ Het & Breast & Pathogenic & $\mathrm{CI}$ & $\begin{array}{l}\text { DM } \\
\text { CM041706 }\end{array}$ & rs55770810 & $\begin{array}{l}\begin{array}{l}\text { Delete- } \\
\text { rious }\end{array} \\
\end{array}$ & \begin{tabular}{|l|} 
Probably \\
damaging
\end{tabular} & $\begin{array}{l}\text { Disease } \\
\text { causing }\end{array}$ & Deleterious & 0.002 & 21 \\
\hline E10 & c.4065_4068delTCAA & p.Asn 1355 Lysfs $^{*} 10$ & Frameshift & $2(1 \%)$ Het & Breast & Pathogenic & $\mathrm{CI}$ & $\begin{array}{l}\text { DM } \\
\text { CD941619 }\end{array}$ & rs 80357508 & - & - & - & - & 0.0008 & 22 \\
\hline E10 & c.1224delA & p.Val409* & Nonsense & $1(0.5 \%)$ Het & Ovarian & Pathogenic & N/A & $\begin{array}{l}\text { DM } \\
\text { CD159317 }\end{array}$ & rs879255320 & - & - & - & - & N/A & 23 \\
\hline E19 & c. $5224 \mathrm{C}>\mathrm{T}$ & p.Gln $1742^{*}$ & Nonsense & $1(0.5 \%)$ Het & Ovarian & Pathogenic & N/A & $\begin{array}{l}\text { DM } \\
\text { CM1211123 }\end{array}$ & N/A & - & - & - & - & N/A & 24 \\
\hline E3 & c. $121 \mathrm{C}>\mathrm{T}$ & p.His41Tyr & Missense & $2(1 \%) \mathrm{Het}$ & Breast & $\mathrm{N} / \mathrm{A}$ & N/A & $\begin{array}{l}\text { DM } \\
\text { CM1712699 }\end{array}$ & N/A & \begin{tabular}{|l|}
$\begin{array}{l}\text { Delete- } \\
\text { rious }\end{array}$ \\
\end{tabular} & \begin{tabular}{|l|} 
Probably \\
damaging
\end{tabular} & $\begin{array}{l}\text { Disease } \\
\text { causing }\end{array}$ & Deleterious & N/A & 25 \\
\hline \multicolumn{16}{|c|}{$B R C A 2$ : pathogenic variants } \\
\hline E10 & c.2254_2257delGACT & p.Asp752Phefs*19 & Frameshift & $2(1 \%) \mathrm{Het}$ & Breast & Pathogenic & CI & DM CD023496 & rs80359326 & - & - & - & - & N/A & 28 \\
\hline E11 & c.5042_5043delTG & p.Val1681Glufs ${ }^{*}$ & Frameshift & $1(0.5 \%) \mathrm{Het}$ & Breast & Pathogenic & $\mathrm{CI}$ & \begin{tabular}{|l} 
DM \\
CD082063
\end{tabular} & rs 80359478 & - & - & - & - & N/A & 30 \\
\hline E11 & c.5351dupA & p.Asn1784Lysfs*3 & Frameshift & $1(0.5 \%)$ Het & Breast & Pathogenic & $\mathrm{CI}$ & \begin{tabular}{|l|} 
DM \\
CI004026
\end{tabular} & rs80359508 & - & - & - & - & N/A & 23 \\
\hline E11 & c.6634_6637delTGTT & p.Cys2212Leufs*16 & Frameshift & $2(1 \%)$ Het & Breast & Pathogenic & N/A & \begin{tabular}{|l} 
DM \\
CD077812
\end{tabular} & rs397507871 & - & - & - & - & N/A & 24 \\
\hline E11 & c.6224_6225delAA & p.Lys2075Serfs $* 2$ & Frameshift & $1(0.5 \%) \mathrm{Het}$ & Ovarian & N/A & N/A & N/A & N/A & - & - & - & - & N/A & Novel \\
\hline $\mathrm{E} 21$ & c. $8696 \mathrm{~A}>\mathrm{G}$ & p.Gln2899Arg & Missense & $1(0.5 \%) \mathrm{Het}$ & Breast & N/A & N/A & N/A & N/A & \begin{tabular}{|l|}
$\begin{array}{l}\text { Delete- } \\
\text { rious }\end{array}$ \\
\end{tabular} & \begin{tabular}{|l|} 
Probably \\
damaging
\end{tabular} & $\begin{array}{l}\text { Disease } \\
\text { causing }\end{array}$ & Deleterious & N/A & Novel \\
\hline
\end{tabular}

Table 3. Pathogenic BRCA1/BRCA2 variants found in breast/ovarian cancer patients $(\mathrm{n}=200)$. BIC Breast Cancer Information Core, HGMD Human Gene Mutation Database, MAF Minor allele frequency, E Exon, Het Heterozygous, Hom Homozygous, CI Clinically Important, DM Disease causing mutation, N/A Not available.

metastasis at diagnosis. Large proportion (83\%) of them had tumor size of $2 \mathrm{~cm}$ or more at time of diagnosis. HER2 receptor status was positive in $24.5 \%$ and negative in $64.5 \%$ of study participants, while the status was not reported for $3.5 \%$ of the cases (Table 1 ).

Overall BRCA1/BRCA2 mutations. The overall prevalence of recurrent and novel mutations (pathogenic and variants of uncertain significance (VUS)) in BRCA1/ BRCA2 genes was $18 \%$ (Table 2). The prevalence of recurrent pathogenic mutations was $14.5 \%$, while the prevalence of novel mutations (pathogenic and VUS) was $3.5 \%$. Two confirmed novel pathogenic mutations were identified in BRCA2 genes. Regarding site of mutation, 7.5\% (15 patients) had pathogenic mutations in BRCA1 gene and 7\% (14 patients) had pathogenic mutations in $B R C A 2$ gene. Five VUS novel variants in $B R C A 2$ gene were identified in this study. Regarding previously reported VUS, this study detected 6 in BRCA1 genes and 9 in BRCA2 genes (Table 3, Supplementary table 2).

Figure 1 shows mutations in BRCA1/BRCA2 according to patients' selection criteria. Participants with relatives with ovarian cancer had higher prevalence of mutations compared with other groups. Regression analysis results showed that E cadherin, PR, ER, oestrogen receptors status, laterality and recurrence since baseline were the statistically significant predictors of the presence of $B R C A 1$ mutations. On the other hand, E cadherin, laterality and recurrence since baseline were statistically significant predictors of $B R C A 2$ mutations. 

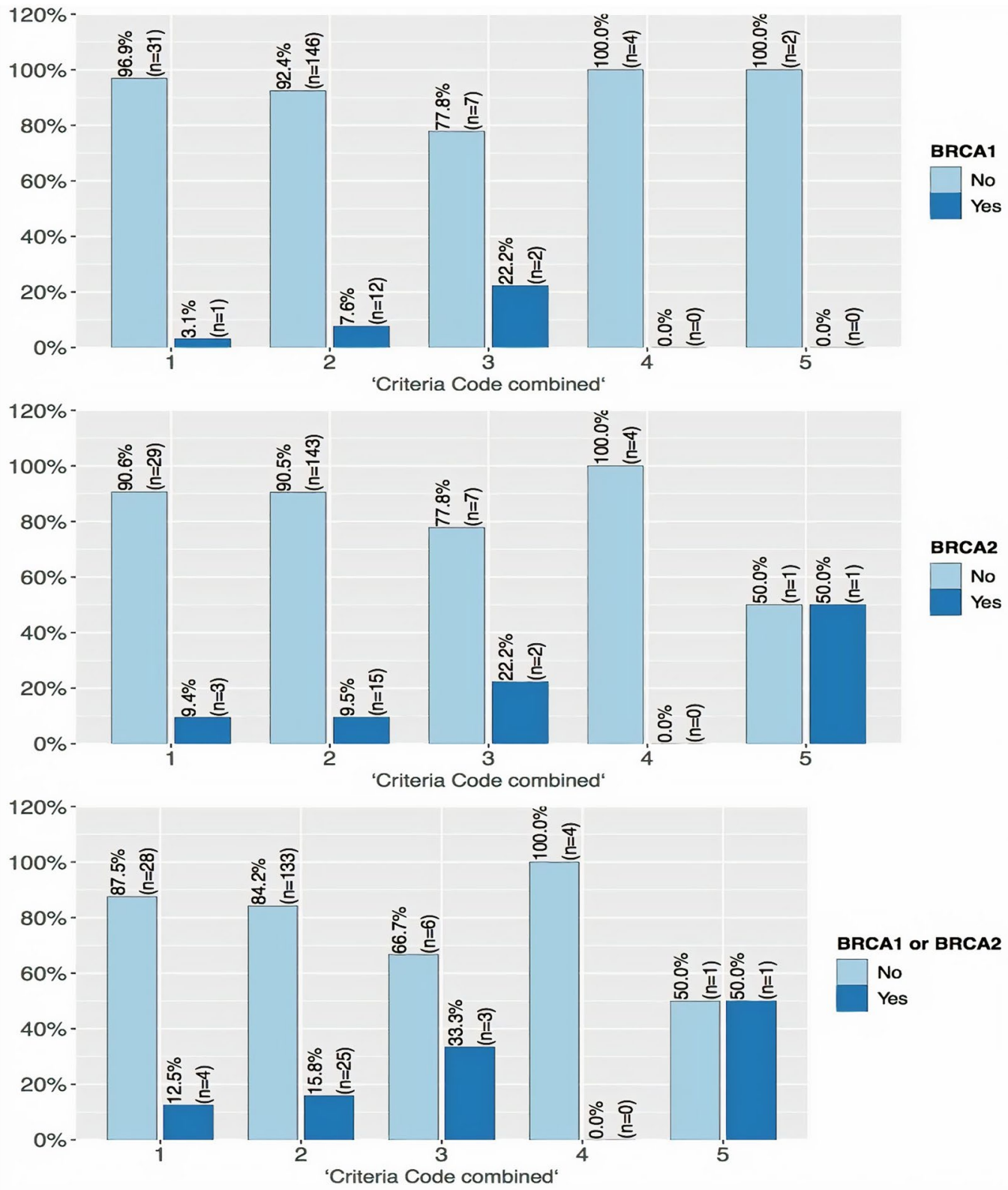

Figure 1. $B R C A 1$ and $B R C A 2$ variants versus inclusion criteria. Top $B R C A 1$ patients, middle $B R C A 2$ patients and bottom $B R C A 1$ and $B R C A 2$ patients. Criteria code combined: 1: Female breast cancer patients younger than the age of 35,2: At least the following female breast cancers only in the family, 3: Families containing one relative with ovarian cancer at any age and, on the same side of the family, 4: Families affected by bilateral cancer (each breast cancer has the same count value as one relative, 5: Families containing male breast cancer at any age and, on the same side of the family.

DNA sequencing and variant analysis. BRCA1 and BRCA2 coding regions and intronic boundaries were amplified. The PCR was performed using the 167 primer pairs. Ion Torrent PGM generated 150 bp average sequence reads length from the sequencer, with 455,768 bp mean target read, $97.5 \%$ Target base coverage at $100 \mathrm{X}, 96.36 \%$ uniformity of base coverage, $90.18 \%$ on targeted region with a $2,738 \mathrm{X}$ average base coverage depth of both genes.

BRCA1/BRCA2 sequencing results revealed 37 different patients with pathogenic and VUS. Seven of those participants had novel variants that do not exist in the literature, diseases and population databases (Table 3 , Supplementary table 2). As listed in Table 3, six BRCA1 variants were pathogenic, two of them were nonsense, one frameshift and three missense variants. In addition, six BRCA1 variants were VUS; four of them were missense variants, one VUS was synonymous, and another was a new splice site change (c.5396-6 T $>$ C) that was present for the first time in our breast cancer patient (Fig. 2, Supplementary table 2). BRCA2 germline variants are presented in Table 3. Two pathogenic BRCA2 variants (p.K2075S fs ${ }^{\star} 2$; c.6224_6225delAA and p.Q2899R; 

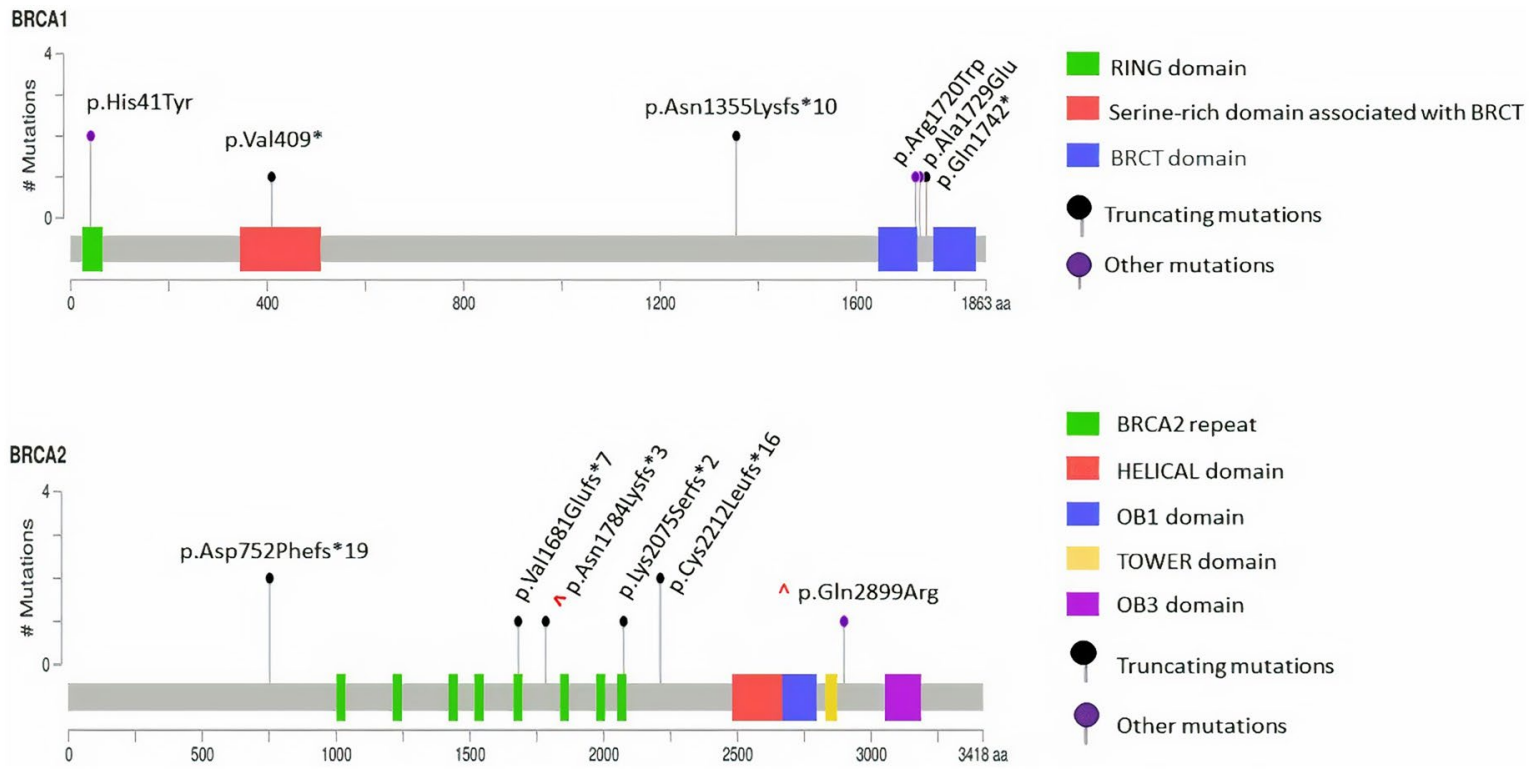

Figure 2. Schematic diagram of BRCA1 and BRCA2 proteins with the position of all identified pathogenic variants. Novel variants are marked with a caret $(\wedge)$.

c.8696A > G) were novel, they are neither registered in the disease nor the population databases. Those two novel pathogenic variants were further validated by Sanger sequencing (Supplementary Fig. $1 \mathrm{a}-\mathrm{b}$ ). Four others recurrent $B R C A 2$ pathogenic variants were identified. They were registered in diseases databases but absent from the population databases gnomAD indicating they are rare variants (Fig. 2 and Table 3 ). The duplication variant in BRCA2 (p.N1784K fs ${ }^{\star} 3$; c.5351dupA) was in a homopolymer region and therefore we validated it through Sanger sequencing (Supplementary Fig. 1c). We also found five distinct novel variant in BRCA2 and classified them as VUS (p.S958R; c.2874 T > G, p.I1000L; c.2998A > C, p.H1525L; c.4574A > T, p. = (p.P2883P), c.8649A > T, p.T3204I; c.9611C > T). In addition, our cohort had nine variants that were classified as VUS (Supplementary table 2). However, the founder variants of $B R C A 1$ (c.185delAG, BRCA1 c.5382insC), and BRCA2 (c.6174delT) in the Ashkenazi Jewish population, were absent in the current cohort, indicating that $B R C A$ variants vary among different populations ${ }^{18}$.

\section{Discussion}

This study was set out to identify $B R C A 1 / B R C A 2$ mutations in patients at high risk of familial breast and ovarian cancer using a systematic approach. Our study showed a high prevalence of $18.0 \%$ for positive mutations in $B R C A 1$ ( $7.5 \%)$ or BRCA2 (10.5\%) genes. Two novel pathogenic mutations and five novel VUS variants were identified in this study, in addition to identifications of patients with recurrent pathogenic and VUS mutations. These figures are higher than recent data from neighboring Arab Peninsula of $16 \%$ or from other Asian countries like Japan of $7 \%{ }^{15-19}$. This could be related to our inclusion criteria based on NICE guidelines for being at high risk of familial breast or ovarian cancers.

Out of 12 pathogenic variants detected in this study in both $B R C A 1 / B R C A 2$, five pathogenic variants were reported previously among Caucasian, Western populations and Latin American. These variants including c.5186C > A, c.4065_4068delTCAA in BRCA1 and c.5042_5043delTG, c.5351dupA in BRCA2 ${ }^{20-24}$.Additionally, three pathogenic variants were previously reported among Asia population. Both c.1224delA and c.5224C $>\mathrm{T}$ in $B R C A 1$ and c.6634_6637delTGTT in BRCA2 were originally reported in Japan and China populations ${ }^{25,26}$. However, different recurrent pathogenic variants were previously reported among Arab population. The c.121C $>\mathrm{T}$ pathogenic variant in BRCA land the c.2254_2257delGACT pathogenic variant in BRCA2 were recently reported in Palestinian patients ${ }^{27,28}$. Additionally, the c.5158C $>\mathrm{T}$ pathogenic variant in $B R C A 1$ was previously reported in Morocco and Saudi Arabia ${ }^{13}$. Both c.6224_6225delAA and c.8696A > G variants in BRCA2 have not been described in the BIC database and other databases including HGMD professional and ClinVar. Therefore, they are considered to be novel. It's worth to mention that four variants in both BRCA1 and BRCA2 were common in more than one patient (Table 3$)^{29-32}$.

Positive patients in our study (recurrent or novel mutations) were diagnosed at advanced stages of breast cancer and ovarian cancers, although they are at high risk of familial cancer. This preventable delay is expected to have a major negative impact on morbidity, morbidity, and cost of management. Such patients could have received preventive cancer treatment giving their very strong family history, should they have been tested properly in due time. Establishing the mutational profile in our region will allow directed testing of high-risk families, therefore increasing the benefit while reducing costs. This is an agreement with Laitman et al. who identified several highly-recurring mutations in the region ${ }^{13}$.

Our participants presented with breast cancer at an early age with a mean of $43.7 \pm 2.7$. Breast cancer at an early age is more likely to be associated with an increased familial risk, particularly in women with a germline 
$B R C A 1$ mutation $^{33}$. In a study of women with breast cancer diagnosed before age 30, BRCA1/BRCA2 and TP53 mutations were found in about half who had strong family histories of breast cancer and in less than $10 \%$ of women with non-familial breast cancer ${ }^{34}$. A young age at diagnosis for breast cancer patients in Jordan and the Arab world suggests a high probability of familial predisposition. A systematic review on age at diagnosis for breast cancer in the Arab world revealed that average age was $48(\mathrm{SD}=2.8)$, range 43-52, median 48.5 and mode 45. These figures are based on data from 28 articles that included years amongst the 7455 patients. The percentage of patients that were younger than 50 years old was reported in 11 articles from 8 countries and included 5144 patients; $65.5 \%(\mathrm{SD}=11$ ) who were less than 50 years old (range $49-78 \%$, median $=66 \%)$. It is recommended that further research to be conducted among young breast and/or ovarian cancer patients in the Arab world to examine the prevalence of known mutations and to search for new potential pathogenic mutations.

Identification of mutations in BRCA1/BRCA2 genes leading to familial breast cancer has had a major impact on prevention of breast and ovarian cancer through development of clinical practice guidelines for management and referral systems for women with a personal or family history of breast or ovarian cancer ${ }^{35}$. Results from some countries (e.g. U.K., France and Canada) and specific cities (Manchester, Marseilles, and Montreal) provided excellent examples of the clinical and economic effectiveness of these preventive services ${ }^{36}$. Management of patients start as early as the age of 25 with clear guidelines for referral and management ${ }^{37}$. In many developing countries, including Jordan, this approach has not been put in place ${ }^{38}$. It has been well-reported in previous studies that positive carriers for $B R C A 1 / B R C A 2$ have high cumulative risk estimates for developing breast cancer reaching $72 \%$ for $B R C A 1$ carriers and $69 \%$ for $B R C A 2$ carrier by age of $80^{5}$. These patients could undergo preventive measures following timely identification of mutations, reducing morbidity and mortality arising from breast and ovarian cancers.

\section{Conclusion}

This study assessed the prevalence of reported mutations and identified novel mutations for BRCA1/BRCA2 genes. Giving the high prevalence of positive mutations in BRCA1/BRCA2 genes, it is essential to establish a service for familial breast and ovarian cancer service in Jordan and the MENA region, that includes genetic counselling and early genetic screening.

\section{Data availability}

All relevant data is available through this manuscript and additional files.

Received: 29 May 2020; Accepted: 2 September 2020

Published online: 16 October 2020

\section{References}

1. Bray, F. et al. Global cancer statistics 2018: GLOBOCAN estimates of incidence and mortality worldwide for 36 cancers in 185 countries. CA Cancer J. Clin. 68, 394-424 (2018).

2. Al-Tarawneh, M., Khatib, S. \& Arqub, K. Cancer incidence in Jordan, 1996-2005. Eastern Mediterranean Health J. 16, 9 (2010).

3. Rhei, E. et al. Molecular genetic characterization of BRCA1-and BRCA2-linked hereditary ovarian cancers. Cancer Res. 58, 31933196 (1998).

4. Kuchenbaecker, K. B. et al. Risks of breast, ovarian, and contralateral breast cancer for BRCA1 and BRCA2 mutation carriers. JAMA 317, 2402-2416 (2017).

5. Rebbeck, T. R. et al. Mutational spectrum in a worldwide study of 29,700 families with BRCA1 or BRCA2 mutations. Hum. Mutat. 39, 593-620 (2018).

6. Smith, S. A., Easton, D. F., Evans, D. G. R. \& Ponder, B. A. J. Allele losses in the region 17q12-21 in familial breast and ovarian cancer involve the wild-type chromosome. Nat. Genet. 2, 128-131 (1992).

7. Thull, D. L. \& Vogel, V. G. Recognition and management of hereditary breast cancer syndromes. Oncologist 9, 13-24 (2004).

8. Narod, S. A. \& Foulkes, W. D. BRCA1 and BRCA2: 1994 and beyond. Nat. Rev. Cancer 4, 665-676 (2004).

9. Abdel-Razeq, H., Al-Omari, A., Zahran, F. \& Arun, B. Germline BRCA1/BRCA2 mutations among high risk breast cancer patients in Jordan. BMC Cancer 18, 152 (2018).

10. Rohlfs, E. M. et al. Direct detection of mutations in the breast and ovarian cancer susceptibility gene BRCA1 by PCR-mediated site-directed mutagenesis. Clin. Chem. 43, 24-29 (1997).

11. Kadouri, L. et al. A novel BRCA-1 mutation in Arab kindred from east Jerusalem with breast and ovarian cancer. BMC Cancer 7 , 14 (2007).

12. Dufloth, R. M. et al. Analysis of BRCA1 and BRCA2 mutations in Brazilian breast cancer patients with positive family history. Sao Paulo Med. J. 123, 192-197 (2005).

13. Laitman, Y. et al. The spectrum of BRCA1 and BRCA2 pathogenic sequence variants in Middle Eastern, North African, and South European countries. Hum. Mutat. 40, e1-e23 (2019).

14. Li, C. I., Malone, K. E. \& Daling, J. R. Differences in breast cancer stage, treatment, and survival by race and ethnicity. Arch. Intern. Med. 163, 49-56 (2003).

15. Hirotsu, Y. et al. Detection of BRCA1 and BRCA2 germline mutations in Japanese population using next-generation sequencing. Mol. Genet. Genom. Med. 3, 121-129 (2015).

16. Trujillano, D. et al. Validation of a semiconductor next-generation sequencing assay for the clinical genetic screening of CFTR. Mol. Genet. Genom. Med. 3, 396-403 (2015).

17. Richards, S. et al. Standards and guidelines for the interpretation of sequence variants: a joint consensus recommendation of the American College of Medical Genetics and Genomics and the Association for Molecular Pathology. Genet. Med. 17, 405-423 (2015).

18. Kim, H. \& Choi, D. H. Distribution of BRCA1 and BRCA2 mutations in Asian patients with breast cancer. J. Breast Cancer 16, 357-365 (2013).

19. Alhuqail, A.-J. et al. High prevalence of deleterious BRCA1 and BRCA2 germline mutations in Arab breast and ovarian cancer patients. Breast Cancer Res. Treat. 168, 695-702 (2018).

20. Futreal, P. A. et al. BRCA1 mutations in primary breast and ovarian carcinomas. Science 266, 120-122 (1994).

21. Abkevich, V. et al. Analysis of missense variation in human BRCA1 in the context of interspecific sequence variation. J. Med. Genet. 41, 492-507 (2004). 
22. Friedman, L. S. et al. Confirmation of BRCA1 by analysis of germline mutations linked to breast and ovarian cancer in ten families. Nat. Genet. 8, 399-404 (1994).

23. Verhoog, L. C. et al. Survival in hereditary breast cancer associated with germline mutations of BRCA2. J. Clin. Oncol. 17, 33963402 (1999).

24. Ang, P. et al. BRCA1 and BRCA2 mutations in an Asian clinic-based population detected using a comprehensive strategy. Cancer Epidemiol. Prev. Biomark. 16, 2276-2284 (2007).

25. Nakamura, S. et al. Prevalence and differentiation of hereditary breast and ovarian cancers in Japan. Breast Cancer 22, 462-468 (2015).

26. Cao, W., Wang, X., Gao, Y., Yang, H. \& Li, J.-C. BRCA1 germ-line mutations and tumor characteristics in eastern Chinese women with familial breast cancer. Anatomical Rec. 296, 273-278 (2013).

27. Lolas Hamameh, S. et al. Genomic analysis of inherited breast cancer among Palestinian women: Genetic heterogeneity and a founder mutation in TP53. Int. J. Cancer 141, 750-756 (2017).

28. El-Harith, E.-H.A., Abdel-Hadi, M. S., Steinmann, D. \& Dork, T. BRCA1 and BRCA2 mutations in breast cancer patients from Saudi Arabia. Saudi Med. J. 23, 700-704 (2002).

29. Seymour, I. J. et al. Results of a population-based screening for hereditary breast cancer in a region of North-Central Italy: contribution of BRCA1/2 germ-line mutations. Breast Cancer Res. Treat. 112, 343-349 (2008).

30. Machackova, E. et al. Spectrum and characterisation of BRCA1 and BRCA2 deleterious mutations in high-risk Czech patients with breast and/or ovarian cancer. BMC Cancer 8, 140 (2008).

31. Maistro, S. et al. Germline mutations in BRCA1 and BRCA2 in epithelial ovarian cancer patients in Brazil. BMC Cancer 16, 934 (2016).

32. Tram, E., Savas, S. \& Ozcelik, H. Missense variants of uncertain significance (VUS) altering the phosphorylation patterns of BRCA1 and BRCA2. PLoS ONE 8, e62468 (2013).

33. Antoniou, A. et al. Average risks of breast and ovarian cancer associated with BRCA1 or BRCA2 mutations detected in case series unselected for family history: a combined analysis of 22 studies. Am. J. Hum. Genet. 72, 1117-1130 (2003).

34. Lalloo, F. et al. BRCA1, BRCA2 and TP53 mutations in very early-onset breast cancer with associated risks to relatives. Eur. J. Cancer 42, 1143-1150 (2006).

35. Weitzel, J. N., Blazer, K. R., MacDonald, D. J., Culver, J. O. \& Offit, K. Genetics, genomics, and cancer risk assessment: state of the art and future directions in the era of personalized medicine. CA Cancer J. Clin. 61, 327-359 (2011).

36. Cm, J.-R. et al. Women's Attitudes Toward Preventive Strategies for Hereditary Breast or Ovarian Carcinoma Differ From One Country to Another: Differences Among English, French, and Canadian Women. Cancer vol. 92 https://pubmed.ncbi.nlm.nih. gov/11550171/ (2001).

37. Forbes, C., Fayter, D., de Kock, S. \& Quek, R. G. A systematic review of international guidelines and recommendations for the genetic screening, diagnosis, genetic counseling, and treatment of BRCA-mutated breast cancer. Cancer Manag. Res. 11, 2321 (2019).

38. Ahmad Abu-Helalah, M. et al. Delay in presentation, diagnosis, and treatment for breast cancer patients in Jordan. Breast J. 22, 213-217 (2016).

\section{Acknowledgments}

We would like to thank Mutah University and University of Jordan for their generous support to the project. Also, we would like to Deanship of Research and Presidency of Mutah University for their great support to the project and continuous encouragement for R\&D in Jordan. Finally, we would like to thank the research coordinators et al.-Bashir Hospital and at the Stem Cell therapy for their valuable contribution to the project.

\section{Author contributions}

M.A.H: Principal Investigator, write the proposal and design the protocol. H.J., Analysis and interpretation of data. D.A., B.A. and N.D., Analysis and interpretation of genetics data. D.A., Perform the genetics experiments. H.S., statistical analysis of the clinical data. R.M., Check inclusion criteria for eligible subjects and invite them for participants in the study, local Principal Investigator at AlBashir Hospital. A.W.: Project supervisor and mentor for all research team, review and edit the proposal and protocol. M.A.H., B.A., D.A. and H.J. write the manuscript and all authors participate in reviewing and editing of the manuscript

\section{Funding}

Mutah University of Jordan, main sponsor, and University of Jordan, partial sponsor.

\section{Competing interests}

The authors declare no competing interests.

\section{Additional information}

Supplementary information is available for this paper at https://doi.org/10.1038/s41598-020-74250-2.

Correspondence and requests for materials should be addressed to A.A.

Reprints and permissions information is available at www.nature.com/reprints.

Publisher's note Springer Nature remains neutral with regard to jurisdictional claims in published maps and institutional affiliations. 
(c) (i) Open Access This article is licensed under a Creative Commons Attribution 4.0 International cc) License, which permits use, sharing, adaptation, distribution and reproduction in any medium or format, as long as you give appropriate credit to the original author(s) and the source, provide a link to the Creative Commons licence, and indicate if changes were made. The images or other third party material in this article are included in the article's Creative Commons licence, unless indicated otherwise in a credit line to the material. If material is not included in the article's Creative Commons licence and your intended use is not permitted by statutory regulation or exceeds the permitted use, you will need to obtain permission directly from the copyright holder. To view a copy of this licence, visit http://creativecommons.org/licenses/by/4.0/.

(C) The Author(s) 2020 